\title{
Unilateral Nail Clubbing in a Hemiparetic Patient
}

\author{
Geraldine Cheyana Ranasinghe, MD; Omar Ibrahim, MD; Christine B. Warren, MD
}

\section{PRACTICE POINTS}

- Unilateral nail changes can be limited to hemiplegic or hemiparetic limbs.

- Lowered pulse oximetry reading only affecting the hemiparetic side demonstrates the possible connection between hypoxia and nail clubbing in this patient population.

\section{To the Editor:}

Few cases of unilateral nail changes affecting only the hemiplegic side after a stroke have been reported. We present a case of acquired unilateral nail clubbing and longitudinal melanonychia in a hemiparetic patient.

A 79-year-old Black man with a history of smoking and stroke presented with concerns of discoloration of the fingernails. His medical history was notable for congestive heart failure; hypertension; diabetes mellitus; hypercholesterolemia; and stroke 11 years prior, which resulted in right-sided hemiparesis. Physical examination revealed longitudinal, even hyperpigmentation of several fingernails on the hands, in addition to whitening of the nail beds, sparing the tips (Terry nails). Clubbing was noted only on the fingernails of the right hand; the fingernails of the left hand exhibited normal curvature (Figure). Pulse oximetry was conducted and demonstrated the following readings: unaffected left index finger, 98\%; unaffected left middle finger, 100\%; affected right index finger, $95 \%$; and affected right middle finger, $97 \%$. The patient
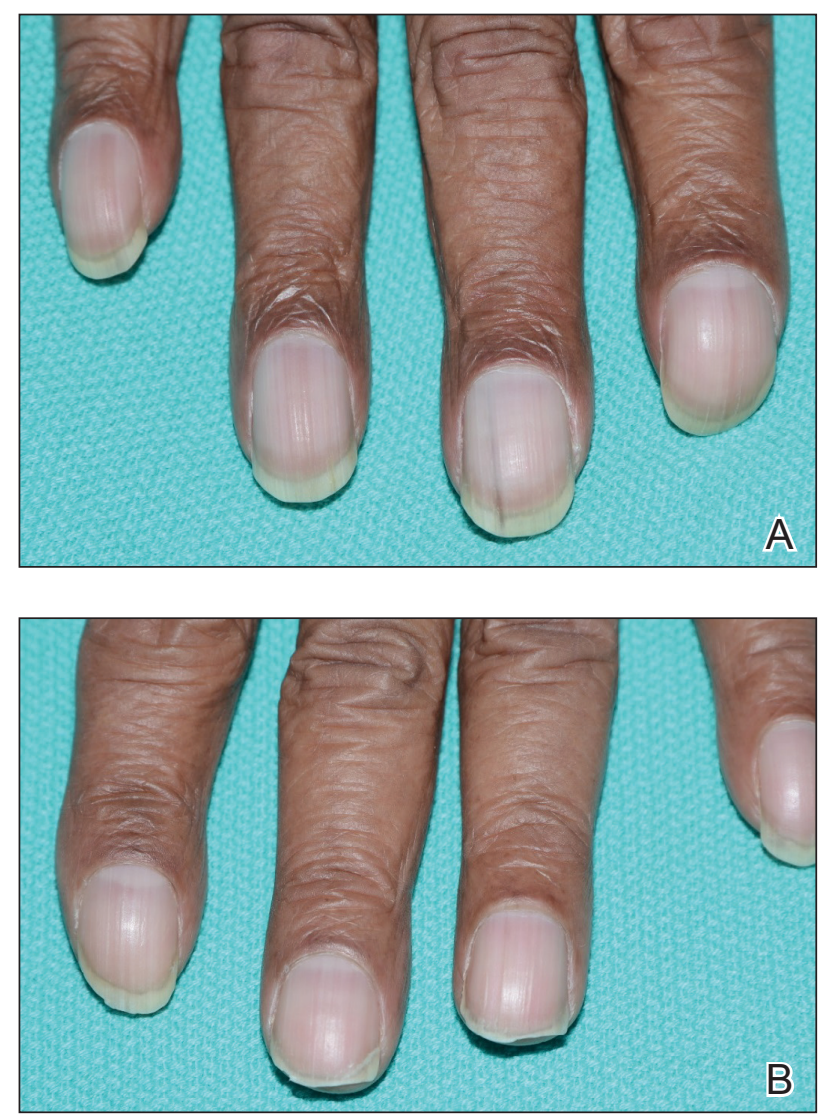

A, Fingernails of the right hand exhibited marked clubbing, causing patient difficulty in trimming nails. B, Fingernails of the left hand exhibited normal curvature.

From the Department of Dermatology, Cleveland Clinic Foundation, Ohio.

The authors report no conflict of interest.

Correspondence: Geraldine Cheyana Ranasinghe, MD, 9500 Euclid Ave, Cleveland, OH 44195 (Ranasig@ccf.org).

doi:10.12788/cutis.0185 
was diagnosed with benign longitudinal melanonychia secondary to ethnic variation, Terry nails without underlying anemia or hypoalbuminemic state, and unilateral right-sided clubbing of the fingernails in the setting of right-sided hemiparesis.

Prior reports have documented the occurrence of nail pathologies after stroke and affecting hemiplegic limbs. Unilateral digital nail clubbing following a stroke was first reported in 1975'; 2 reports concluded clubbing developed in all digits affected by the stroke, and the severity of clubbing was associated with the duration of the stroke. ${ }^{1,2}$ One study noted longitudinal reddish striation, Neapolitan nails, and unilateral clubbing more commonly in hemiplegic patients. ${ }^{3}$ Longitudinal reddish striation was the most frequent condition observed in this population, always affecting the entire thumbnail of the hemiplegic limb. ${ }^{3}$ A similar report observed clubbing only on the fingernails of the hemiplegic side. ${ }^{4}$

Digital clubbing describes an exaggerated nail curvature and bulbous overgrowth of the fingertips due to an expansion of connective tissue between the nail plate and the nail bed. ${ }^{3,5}$ Clubbed fingers are found in various chronic conditions affecting the heart, lungs, and liver. Although the pathogenesis of clubbing remains unknown, many hypothesize that it is a state of proliferation in response to digital hypoxia. ${ }^{5}$ Fittingly, our patient exhibited a relative hypoperfusion of the clubbed fingers in comparison to the unaffected side.

This case provides additional support for the phenomenon of unilateral nail changes limited to hemiplegic or hemiparetic limbs. The unique presentation of longitudinal melanonychia, clubbing, and a lowered pulse oximetry reading only affecting the hemiparetic side demonstrates the possible connection between hypoxia and nail clubbing in this patient population.

\section{REFERENCES}

1. Denham $\mathrm{M}$, Hodkinson $\mathrm{H}$, Wright B. Unilateral clubbing in hemiplegia. Gerontology Clin (Basel). 1975;17:7-12.

2. Alveraz A, McNair D, Wildman J, et al. Unilateral clubbing of the fingernails in patients with hemiplegia. Gerontology Clin (Basel). 1975;17:1-6.

3. Siragusa M, Schepis C, Cosentino F, et al. Nail pathology in patients with hemiplegia. Br J Dermatol. 2001;144:557-560.

4. Gül Ü, Çakmak S, Özel S, et al. Skin disorders in patients with hemiplegia and paraplegia. J Rehabil Med. 2009;41:681-683.

5. Sarkar M, Mahesh D, Madabhavi I. Digital clubbing. Lung India. 2012;29:354-362. 